Methods Monthly information on employment status of the Dutch population $(n=4,999,947)$ between 16 and 66 year from 2001 to 2015 was used to estimate working life courses. Across educational groups monthly transitions between paid employment and non-employment states were calculated with a Markov model with transitional probabilities. Using this multistate model (R-package mstate) the WLE and WYL due to disability benefits and other nonemployment states were estimated, stratified by educational groups.

Results Despite starting in paid employment much earlier, low educated men and low educated women had a 4.17 (men) and 9.50 (women) years lower WLE at age 16 than high educated men and women. Among low educated men 3.59 WYL were due to disability benefit compared to $0.78 \mathrm{WYL}$ among high educated men, resulting in a WYL gap 2.81 years. Low educated women had 3.47 WYL due to disability benefit compared to 1.38 WYL among high educated women introducing a WYL gap of 2.09. Educational inequalities in premature death added to this WYL gap another 0.7 years among men and 0.3 years among women.

Conclusions The working life course showed large educational differences. A considerable amount of the lost working time is health-related due to disability benefits and premature death. In comparison to high educated workers, those with a low educational level lose a substantial part of their working life due to disability benefit, unemployment, and no income. The metrics of WLE and WYL provide useful insights into the life-course perspective of working careers.

\section{D.3 WORKING CONDITIONS AND HEALTH BEHAVIOUR AS CAUSES OF EDUCATIONAL INEQUALITIES IN SELF- RATED HEALTH: AN INVERSE ODDS WEIGHTING APPROACH}

\begin{abstract}
${ }^{1}$ Jolinda Schram*, ${ }^{1,2}$ Joost Oude Groeniger, ${ }^{1}$ Merel Schuring, ${ }^{3}$ Karin Proper, ${ }^{3}$ Sandra van Oostrom, 'Suzan Robroek, 'Alex Burdorf. 'Erasmus Medical Centre Rotterdam, Department of Public Health, Rotterdam, Netherlands; ${ }^{2}$ Department of Public Administration and Sociology, Erasmus University, Rotterdam, Netherlands; ${ }^{3}$ Centre for Nutrition, Prevention and Health Services, National Institute for Public Health and the Environment (RIVM), Bilthoven, Netherlands
\end{abstract}

\subsection{6/OEM-2019-EPI.180}

Background This study aims to estimate to what extent working conditions and health behaviours mediate the increased risk of low educated workers to report a poor health.

Methods Respondents of the longitudinal Survey of Health, Ageing, and Retirement in Europe (SHARE) in 18 European countries were selected aged between 50 years and 64 years, in paid employment at baseline and with information on education and self-rated health $(n=15,126)$. Health behaviours and physical and psychosocial work characteristics were measured at baseline, while self-rated health was measured at 2 year follow up. We used loglinear regression models and Inverse Odds Weighting causal mediation analysis to estimate the total effect of low education on self-rated health and to decompose the effect into natural direct (NDE) and natural indirect effects (NIE).

Results Lower educated workers were more likely to be in poor health compared to higher educated workers. The total effect of low education on self-rated health was $\mathrm{RR}=1.81$ [95\% CI 1.66-1.97]. For work conditions, having a physical demanding job was the strongest mediator, followed by lack of job control and lack of job rewards. NIE through working conditions was $\mathrm{RR}=1.16$ [95\% CI $1.06-$ 1.25], explaining about $30 \%$ of educational inequalities in self-rated health. For health behaviour, body mass index and alcohol were the strongest mediators, followed by smoking. NIE though health behaviour was $\mathrm{RR}=1.14$ [95\% CI 1.071.20], explaining about $27 \%$ of educational inequalities in self-rated health.

Conclusions Preventive interventions focusing on reducing physical work demands as well as improving health behaviour may contribute to reducing educational inequalities in selfrated health among workers in Europe.

\section{D.4 HEALTH INEQUALITIES AMONG WORKING POPULATION OF LATIN AMERICA AND THE CARIBBEAN}

\begin{abstract}
1,2Michael Silva-Peñaherrera* 1,3,4,5 María Lopez-Ruiz, 1,2Pamela Merino-Salazar, ${ }^{2}$ Antonio Goméz-García, ${ }^{1,3,4}$ Fernando Benavides. 'Center for Research in Occupational Health (CISAL) Universitat Pompeu Fabra (UPF), Barcelona, Barcelona, España; ${ }^{2}$ Research Group on Working Conditions and Occupational Health, International SEK University, Quito, Ecuador; ${ }^{3}$ CIBER Epidemiología y Salud Pública (CIBERESP), España; ${ }^{4}$ MIM (Hospital del Mar Medical Research Institute), Barcelona, España; ${ }^{5}$ Facultad Latinoamericana de Ciencias Sociales, Salamanca, España
\end{abstract}

\subsection{6/OEM-2019-EPI.181}

Background Working and employment conditions are weighty health determinants. Low-income countries are frequently exposed to occupational risks, employment agreements are poorly regulated and a high proportion of the working population are in the informal sector. Studies in Latin American and the Caribbean (LAC) have been hampered by the lack of reliable data available.

Objective To describe and compare the health inequality gap among workers of 20 countries of LAC.

Methods Data have been taken from the last available Working Conditions Survey, National Health Surveys and from official records and national statistical institutes of each country. Three indicators were calculated to estimate health status of the working population (poor-self-perceived health, fatal and non-fatal injuries at work and general mortality within the working-age population), stratified by sex, age, level of study and occupation. Disparity was measured using Keppel index of disparity (ID) and Kuznets relative. Absolute and relative differences were calculated using the best health status country as reference category.

Results Mortality among the working-age population was higher in men and varied from 87.4 deaths per 1000 inhabitants in Chile to over 182.2 in Bolivia. Keppel ID between countries was 0.5 , indicating medium-high disparity. The prevalence of poor self-perceived health was higher in women and revealed a gradient by age, education level and occupation, in which young people in qualified non-manual occupations and high levels of study had lower prevalence. The ID was 0.7 between countries demonstrating high disparity and the range varied from 12.2 in Uruguay to 50.9 in Nicaragua.

Conclusions This study reveals the existence of wide gaps in health among workers in LAC, both within and between countries. The limitations of cross-country comparative data should considered. However, this analysis increases our understanding of the causes of inequalities and provides evidence to establish better public policies. 